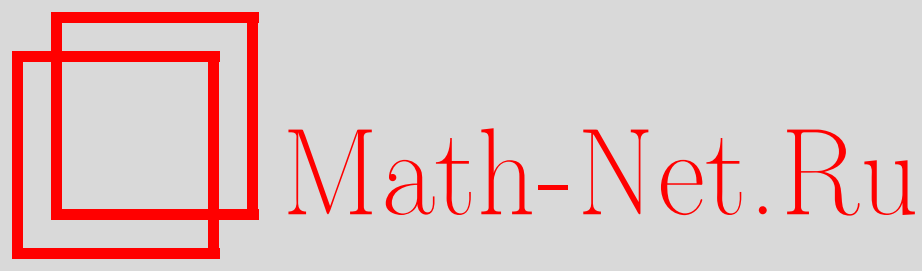

А. Б. Богатырёв, Вещественные мероморфные дифференциалы: язык для описания меронных конфигураций в планарных магнитных наноэлементах, ТMФ, 2017, том 193, номер 1, 162-176

DOI: https://doi.org/10.4213/tmf9301

Использование Общероссийского математического портала Math-Net.Ru подразумевает, что вы прочитали и согласны с пользовательским соглашением http://www . mathnet.ru/rus/agreement

Параметры загрузки:

IP : 18.234 .156 .22

26 апреля 2023 г., 05:29:06

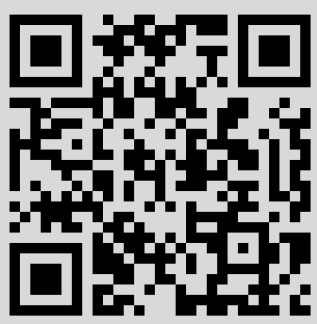




\title{
ВЕЩЕСТВЕННЫЕ МЕРОМОРФНЫЕ ДИФФЕРЕНЦИАЛЫ: ЯЗЫК ДЛЯ ОПИСАНИЯ МЕРОННЫХ КОНФИГУРАЦИЙ В ПЛАНАРНЫХ МАГНИТНЫХ НАНОЭЛЕМЕНТАХ
}

\begin{abstract}
Для описания метастабильных состояний многосвязных плоских ферромагнитных наноэлементов, минимизирующих обменную энергию и не имеющих боковых магнитных зарядов, используется язык вещественных мероморфных дифференциалов из теории поверхностей Клейна. Эти решения все же имеют достаточное количество внутренних степеней свободы, которые можно использовать как параметры Ритца для минимизации других вкладов в общую энергию или как медленные динамические переменные в адиабатическом приближении. Нетривиальная топология самого магнетика приводит к нескольким эффектам, впервые описанным для кольца и наблюдаемым в эксперименте. Объясняются связи между числами топологических особенностей разных видов в магнетике, а также вытекающие из теоремы Абеля ограничения на положения этих особенностей. Использование многозначных дифференциалов Прима приводит к новым меронным конфигурациям, не рассматриваемым в классической работе Гросса.
\end{abstract}

Ключевые слова: спинтроника, планарный наноэлемент, магнитный вихрь, мерон, поверхность Клейна, дифференциал Прима.

DOI: https://doi.org/10.4213/tmf9301

\section{1. ВВЕДЕНИЕ}

Ферромагнетики на наномасштабах содержат в себе удивительный мир взаимодействующих квазичастиц, вихреобразных состояний распределения намагниченности в веществе. Понятия скирмионов, инстантонов и меронов были введены много

Работа поддержана РНФ (грант № 16-11-10349).

* Московский физико-технический институт (государственный университет), Долгопрудный, Московская обл., Россия. E-mail: ab.bogatyrev@gmail.com

${ }^{\dagger}$ Московский государственный университет им. М. В. Ломоносова, Москва, Россия

${ }^{\ddagger}$ Институт вычислительной математики РАН, Москва, Россия 
лет назад в физике высоких энергий, однако они используются и в физике конденсированного состояния, где вихреподобные топологии описывают, например, системы с квантовым эффектом Холла, определенные жидкокристаллические фазы, конденсаты Бозе-Эйнштейна и т. п. Магнитные вихри очень важны для возникающей спинтронной промышленности, где они рассматриваются как перспективные кандидаты на перенос и хранение информации, поскольку обладают размерами всего в несколько нанометров, очень устойчивы и легко управляемы с незначительными затратами энергии. Принимая во внимание промышленные приложения, отметим, что именно плоские конфигурации магнитов становятся особенно интересными.

Гамильтониан, управляющий динамикой магнитного состояния, учитывает довольно много взаимодействий (таких как обменное, магнитодипольное, взаимодействие Дзялошинского-Мори, взаимодействие с внешним полем, магнитостатическую энергию и т. д.), которые образуют определенную иерархию. Среди физиков есть понимание, что в случае планарных ферромагнитов наноразмеров наибольший вклад в поведение состояния вносит обменное взаимодействие. Один из подходов к исследованию магнитных состояний (см. [1] и приведенные там ссылки) состоит в минимизации обменной энергии Гейзенберга и получении тем самым метастабильных состояний, дальнейшая динамика которых определяется следующими по значению членами иерархии гамильтониана.

В настоящей работе мы используем язык вещественных мероморфных дифференциалов из теории поверхностей Клейна [2], [3] для описания метастабильных состояний многосвязного планарного ферромагнитного наноэлемента, минимизирующих обменную энергию и не имеющих боковых магнитных зарядов. Последнее свойство означает минимальность соответствующей части магнитостатической энергии. Такие решения все же имеют достаточно внутренних степеней свободы, которые можно использовать как параметры Ритца для минимизации последующих членов в иерархии энергии или как медленные динамические переменные в адиабатическом приближении. Нетривиальная топология самого магнита приводит к некоторым эффектам, впервые описанным для кольца [4] и наблюдаемым в эксперименте. Здесь мы объясняем топологические ограничения на число особенностей намагниченности разных типов, а также алгебраические ограничения на их положения, вытекающие из теоремы Абеля.

Использование многозначных дифференииалов Прима приводит к новым меронным конфигурациям, которые не рассматривались в основополагающей работе Гросca [5].

\section{2. ПОСТАНОВКА ЗАДАЧИ}

В этом разделе мы кратко формулируем модель Метлова, описывающую магнитные текстуры в плоских наноэлементах и предложенную в работах [1], [6]. Модель основана на допущении определенной иерархии различных вкладов в полную энергию магнетика (таких, как обменная энергия и магнитостатическое взаимодействие). Такая иерархия обычно имеет место в магнитах субмикронных размеров (магнитных наноэлементах). В планарном наномагните, имеющем форму тонкого цилиндра, магнитную текстуру при отсутствии внешнего магнитного поля можно описать при помощи мероморфной функции комплексной переменной, возникающей 
при решении краевой задачи Римана-Гильберта [7]. Эту задачу можно естественно переформулировать в терминах вещественных мероморфных дифференциалов, которые и являются основным предметом настоящей работы.

Обменная энергия двумерного наномагнетика, заполняющего плоскую область $\Omega$, имеет вид [8]

$$
E[m]=\int_{\Omega} \sum_{a=1}^{3}\left|\nabla m_{a}\left(x_{1}, x_{2}\right)\right|^{2} d \Omega,
$$

где (нормированное) распределение намагниченности $m\left(x_{1}, x_{2}\right)=\left(m_{1}, m_{2}, m_{3}\right)$ - это гладкое отображение из области $\Omega$ в единичную сферу: $|m|^{2}:=\sum_{a} m_{a}^{2}=1$. Поскольку и область определения, и множество значений отображения намагниченности $m$ являются поверхностями, возникает искушение использовать в этой задаче язык комплексных переменных. Для этого вводят комплексную координату $z:=x_{1}+i x_{2}$, изменяющуюся в области $\Omega$, а также стереографическую проекцию сферы на комплексную плоскость переменной $w$ :

$$
m_{1}+i m_{2}=\frac{2 w}{1+w \bar{w}}, \quad m_{3}=\frac{1-w \bar{w}}{1+w \bar{w}} .
$$

В новых терминах обменная энергия может быть выражена так:

$$
E[w]=\int_{\Omega} \frac{8}{(1+w \bar{w})^{2}}\left(\left|w_{z}\right|^{2}+\left|\bar{w}_{z}\right|^{2}\right) d \Omega
$$

где мы использовали обозначение Виртингера для комплексного дифференцирования $\partial / \partial z$; заметим, что функция $w(z)$ может не быть ни аналитической, ни антианалитической. Уравнение Эйлера-Лагранжа для последнего функционала принимает вид

$$
w_{z \bar{z}}=\frac{2 \bar{w}}{1+w \bar{w}} w_{z} w_{\bar{z}}
$$

Общее решение этого уравнения неизвестно (во всяком случае, автору). Два широких класса локальных решений - это инстантоны $w=f(z)$, введенные Белавиным и Поляковым [9], и мероны (или полуинстантоны) $w=f(z) /|f(z)|$, открытые Гроссом [5]. Здесь $f(z)$ есть (анти)голоморфная функция переменной $z$. Легко показать, что общее решение уравнения (4) с намагниченностью $m$, лежащей в плоскости магнетика, является в точности меронным. Действительно, в последнем случае намагниченность имеет вид $m(z)=(\cos \Phi, \sin \Phi, 0)$, и обменная энергия (1) становится просто интегралом Дирихле для вещественнозначной функции $\Phi(z)$ :

$$
E[m]=\int_{\Omega}\left(\sin ^{2} \Phi+\cos ^{2} \Phi\right)|\nabla \Phi|^{2} d \Omega .
$$

Это означает, что функция $\Phi(z)$ гармонична и локально является мнимой частью некоторой голоморфной функции $h(z)$. Так получаем (локально) меронное представление для решения $w(z)$ с функцией $f=e^{h(z)}$.

Итак, если дана любая мероморфная функция $f$ в области, мы можем построить или инстантон, или мерон. Заметим, что мерон, соответствующий функции $f(z)$, имеющей нули или полюсы, обладает бесконечной энергией. В этой связи Гросс [5] 

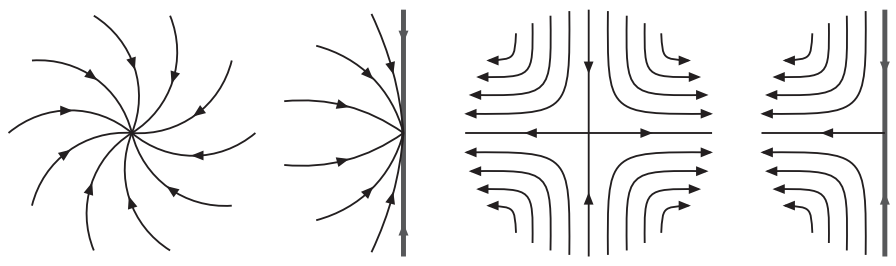

Рис. 1. Простые вихри (соответствуют полюсам $d \xi$ ) и антивихри (или седла, нули $d \xi)$ внутри области и на ее границе.

писал: “Конфигурации с бесконечным действием могут быть физически значимыми, если действие логарифмически расходится в зависимости от объема". В нашем случае энергия расходится как логарифм объема малой окрестности нуля или полюса. При заданной мероморфной функции $f$ также рассматривают [1], [5] имеющую конечную энергию смесь двух основных решений вида

$$
w(z)= \begin{cases}f(z) / E_{1}, & |f(z)| \leqslant E_{1}, \\ f(z) /|f(z)|, & E_{1} \leqslant|f(z)| \leqslant E_{2} \\ f(z) / E_{2}, & E_{2} \leqslant|f(z)|\end{cases}
$$

или

$$
w(z)= \begin{cases}f(z) / E_{1}, & |f(z)| \leqslant E_{1}, \\ f(z) /|f(z)|, & E_{1} \leqslant|f(z)| \leqslant E_{2} \\ E_{2} / \overline{f(z)}, & E_{2} \leqslant|f(z)|\end{cases}
$$

Решение такого вида непрерывное, но не гладкое: его нормальная производная рвется вдоль линий $z:|f(z)|=E_{1},|f(z)|=E_{2}$, и выражение (4), вычисленное на таком составном решении, приобретает источники, сосредоточенные на этих линиях.

Чтобы получить физически значимое глобальное решение, нужно снабдить его подходящими краевыми условиями. Условие непротекания - вектор $w(z)$ почти везде направлен вдоль границы [1], [4], [10] - не конфликтует с уравнением (4) и моделирует доменные стенки на границе. С математической точки зрения это дает однородную задачу Римана-Гильберта [7], с физической - мы устраняем таким образом боковые магнитные заряды [8], а следовательно, и их магнитостатическую энергию. Заметим, что минимизация функционала обменной энергии (3) требует других граничных условий, а именно условий Неймана $w_{z} d z-w_{\bar{z}} d \bar{z}=0$ на границе.

Суммируя вышесказанное, мы можем извлечь разумную математическую постановку: найти мероморфные в области $\Omega$ функции $f$ такие, что дифференциал $d \xi:=d z / f(z)$ является вещественным на границе области.

Это в точности задача описания всех вещественных мероморфных дифференииалов в области. Переход от функций $f$ к дифференциалам $d \xi$ раскрывает конформную инвариантность первоначальной задачи. В самом деле, конформное отображение переносит вещественные дифференциалы из одной области в другую (с теми 
же конформными модулями), тем самым сохраняя не только решения дифференциального уравнения, но и краевое условие непротекания. Нули и полюсы дифференциала (которые могут лежать и на границе магнетика) соответствуют топологическим особенностям намагниченности (см. рис. 1). Согласно Гельмгольцу вещественные мероморфные дифференциалы также описывают (локально потенциальные) стационарные движения идеальной жидкости с возможными источниками и стоками в плоской области, что дает еще одну связь между предметами гидродинамики и (микро)магнетизма.

\section{3. ДУБЛЬ ШОТТКИ ПЛОСКОЙ ОБЛАСТИ И ВЕЩЕСТВЕННЫЕ ДИФФЕРЕНЦИАЛЫ}

Пусть $\Omega$ - конечносвязная область на плоскости с гладкими границами. Ее дубль Шоттки - это замкнутая риманова поверхность рода, на единицу меньшего упомянутого числа связности, обозначаемая $\mathcal{D} \Omega$ и получаемая следующим образом [2]. Возьмем две копии области и отождествим компоненты их границ естественным образом. Если $z$ - локальная координата на одной копии области $\Omega$, то $\bar{z}$ будет локальной координатой на второй копии. Дубль $\mathcal{D} \Omega$ допускает антиконформную инволюиию (также называемую отражением) $\tau$, состоящую в смене двух копий области. Неподвижные точки этого отражения - это в точности границы первоначальной области, называемые теперь вещественными овалами дубля. Отражение $\tau$ естественно действует на закнутых кривых поверхности, расщепляя тем самым ее 1-гомологии на четные (сохраняющиеся при отражении) и нечетные (меняющие знак) циклы [2], [11]. Другим естественным действием антиконформной инволюции является ее действие на пространствах голоморфных и мероморфных дифференциалов, состоящее в вызванной отражением замене координат с последующим комплексным сопряжением. Указанное действие также расщепляет дифференциалы на вещественные, остающиеся неизменными, и мнимые, соответственно меняющие знак при таком действии [2], [3]. Вещественными являются ровно те дифференциалы, сужение которых на любой вещественный овал является вещественным. Несложно проверить, что интегрирование вещественных и мнимых дифференциалов по четным циклам дает соответственно вещественный и мнимый ответ, в то время как спаривание их с нечетными циклами является, наоборот, мнимым и вещественным соответственно [11].

Описание всех вещественных дифференциалов является в некотором смысле тривиальной задачей: возьмем любой мероморфный дифференциал на дубле $\mathcal{D} \Omega$ и симметризуем его по отношению к индуцированному действию отражения $\tau$.

\section{1. Примеры.}

Пример 1. Вещественные мероморфные дифференциалы в двусвязной области можно перечислить следующим образом. Любое топологическое кольцо конформно эквивалентно расширенной комплексной плоскости $\widehat{\mathbb{C}}=\mathbb{C} \cup\{\infty\}$ переменной $z$ с двумя симметричными вещественными щелями, скажем $\pm[1,1 / k]$, здесь $0<k<1-$ конформный модуль области. Дубль такой области эквивалентен эллиптической кривой

$$
(z, w) \in \mathbb{C}^{2}: \quad w^{2}=\left(z^{2}-1\right)\left(k^{2} z^{2}-1\right)
$$


с антиконформной инволюцией $\tau(z, w)=(\bar{z},-\bar{w})$. Мероморфный дифференциал на этой поверхности имеет вид

$$
d \omega(z, w)=\left(R_{1}(z)+w R_{2}(z)\right) d z
$$

с рациональными функциями $R_{1}, R_{2}$ переменной $z$. Среди них вещественными являются дифференциалы, удовлетворяющие условию $d \omega(\bar{z},-\bar{w})=\overline{d \omega(z, w)}$, т. е. с вещественными рациональными функциями $R_{1}(z)$ и $i R_{2}(z)$.

Пример 2. Трехсвязные области можно рассмотреть схожим образом: любые топологические штаны конформно эквивалентны расширенной комплексной плоскости с тремя выброшенными вещественными отрезками, например $\bigcup_{j=1}^{3}\left[e_{2 j-1}, e_{2 j}\right]$, где три точки из $e_{1}<e_{2}<\cdots<e_{6}$ можно нормировать произвольно, три других можно считать конформными модулями штанов. Дублем этой области является кривая рода 2

$$
(z, w) \in \mathbb{C}^{2}: \quad w^{2}=\prod_{j=1}^{6}\left(z-e_{j}\right)
$$

с отражением $\tau$, как в предыдущем примере, и вещественные дифференциалы имеют вид (5) с вещественными рациональными функциями $R_{1}(z)$ и $i R_{2}(z)$, как и в предыдущем примере.

Пример 3. Вещественные дифференциалы можно также получить в терминах тета-функций. Кольцо $1 \leqslant|z| \leqslant R$ мы представим как фактор вертикальной полосы $0 \leqslant \operatorname{Re}(u) \leqslant 1 / 2$ по группе ее сдвигов, порожденной мнимой величиной $i T$, где $T=\pi / \ln (R)>0$. Соответствие двух моделей кольца дается явной формулой $z(u)=$ $e^{2 \pi u / T}$.

В этой модели можно построить все вещественные мероморфные дифференциалы $d \xi$, инвариантные относительно сдвигов $u \rightarrow u+i T$. Простейший из них это $d \xi=i d u$, все прочие получаются домножением на вещественную на границах полосы мероморфную функцию $h(u)$, обладающую нужной инвариантностью при сдвигах. Принцип симметрии продолжает такую функцию до мероморфной во всей плоскости, в которой она имеет решетку периодов $\mathbb{Z}+i T \mathbb{Z}$. Двоякопериодические функции имеют эффективное представление в терминах эллиптических тета-функций [12]. Выберем представителя (по модулю решетки периодов) $a_{s}^{+}$для каждого нуля функции $h$ и представителя $a_{s}^{-}$для каждого ее полюса. Последние удовлетворяют известному решеточному условию [12]

$$
\sum_{s=1}^{N}\left(a_{s}^{+}-a_{s}^{-}\right) \in \mathbb{Z}+m i T, \quad m \in \mathbb{Z}, \quad N=\operatorname{deg} h(u) .
$$

При данном выборе представителей наша функция пропорциональна функции

$$
h(u)=e^{-2 \pi i m u} \prod_{j=1}^{N} \frac{\theta_{1}\left(u-a_{j}^{+}\right)}{\theta_{1}\left(u-a_{j}^{-}\right)}
$$

где $\theta_{1}(u)=e^{-\pi T / 4} \sin (\pi u)-\cdots$ есть единственная нечетная тета-функция модуля $i T$.

Если множества нулей и полюсов функции $h(u)$ зеркально-симметричны по отношению к мнимой оси, то из симметрий тета-функции можно вывести, что (подходящим образом нормированная) функция $h(u)$ принимает вещественные значения на 


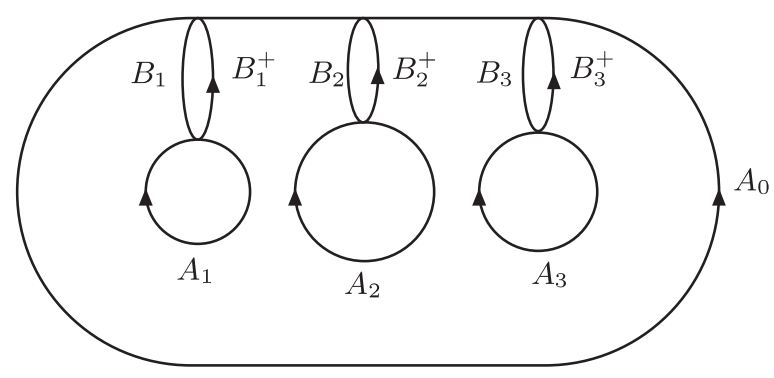

Рис. 2. Базис гомологий на дубле $\mathcal{D} \Omega$ плоской области $\Omega$.

границах полосы: $\{0,1 / 2\}+i \mathbb{R}$. Мы перенесем дифференциал $d \xi=i h(u) d u$ с полосы в концентрическое кольцо с помощью экспоненциального отображения $z(u)$, при этом решеточное условие (7) становится необходимым и достаточным условием существования в кольце вещественного дифференциала с данным набором нулей и полюсов:

1) число нулей равно числу полюсов;

2) общее число нулей и полюсов на каждой границе (полувихрей) целое;

3) сумма азимутов нулей равна сумме азимутов полюсов по модулю $\pi$.

Здесь азимут вычисляется по отношению к центру кольца с точностью до $2 \pi$ и нули с полюсами на границе считаются с весом $1 / 2$.

Авторы работы [4] проверили справедливость последнего ограничения на положения магнитных вихрей в наноэлементах на доступных экспериментальных данных и обнаружили хорошее согласие с экспериментом.

3.2. Гомологии и дифференциалы на дубле Шоттки. Из последнего примера мы узнали, что положения нулей и полюсов вещественного дифференциала в кольце не могут быть произвольными. Тот же эффект наблюдается в областях большей связности, и, по-существу, он связан с теоремой Абеля о дивизорах алгебраических функций [13], [14]. Для объяснения этого явления введем вспомогательные конструкции.

3.2.1. Базис из четных и нечетных циклов. На дубле $(g+1)$-связной области $\Omega$ рассмотрим базис $A_{1}, \ldots, A_{g} ; B_{1}, \ldots, B_{g}$ целых 1-гомологий (замкнутых контуров по модулю некоторой эквивалентности) со стандартной симплектической формой пересечений

$$
A_{j} \circ A_{s}=B_{j} \circ B_{s}=0, \quad A_{j} \circ B_{s}=\delta_{j s}, \quad j, s=1, \ldots, g,
$$

и дополнительно с зеркальной симметрией:

$$
\tau A_{j}=A_{j} ; \quad \tau B_{j}=-B_{j}, \quad j=1, \ldots, g .
$$

Базис с такими свойствами существует, но не является единственным. Например, можно взять естественно ориентированные внутренние границы области $\Omega \subset \mathcal{D} \Omega$ в качестве $A$-циклов. Каждый из них мы соединяем с внешней границей $A_{0} \sim$ $-\sum_{j=1}^{g} A_{j}$ с помощью $g$ попарно разделенных простых дуг $B_{j}^{+}$, которые замыкаем до нечетных циклов на дубле: $B_{j}:=B_{j}^{+}-\tau B_{j}^{+}$(см. рис. 2). 
3.2.2. Голоморфные дифференциалы. Для представления различных теоретико-функциональных объектов на римановой поверхности используют, например, голоморфные и мероморфные дифференциалы. Построим базис голоморфных дифференциалов. В области $\Omega$ существует $g$ так называемых гармонических мер $W_{s}(z)$, т. е. гармонических функций, исчезающих на всех границах области, за исключением одной внутренней границы $A_{s}$, где эта функция равна единице. Сопряженные к ним гармонические функции $H_{s}(z)$ являются многозначными в области, однако дифференциалы $d \omega_{s}:=d\left(W_{s}+i H_{s}\right)$ голоморфны и являются чисто мнимыми на границах области. Последнее обстоятельство позволяет голоморфно продолжить их на замкнутую поверхность $\mathcal{D} \Omega$ как мнимые дифференциалы:

$$
\tau \cdot d \omega_{s}=-d \omega_{s}, \quad s=1, \ldots, g .
$$

Периоды этих дифференциалов следующие:

$$
\int_{B_{s}} d \omega_{j}=-2 \delta_{j s}, \quad \int_{A_{s}} d \omega_{j}=i \Pi_{j s},
$$

где $\Pi_{j s}$ - вещественная симметричная матрица с положительными диагональными и отрицательными внедиагональными элементами, а также с диагональным преобладанием. Ее можно интерпретировать как матрицу емкостей области. В самом деле, $W_{j}$ можно считать напряжением, приводящим к появлению заряда

$$
\int_{A_{s}}\left(\frac{d W_{j}}{d n}\right) d l=\int_{A_{s}}\left(\frac{d H_{j}}{d l}\right) d l=-i \int_{A_{s}} d \omega_{j}=\Pi_{j s}
$$

на граничной компоненте $A_{s}(n$ и $l$ - нормальное и касательное направления к границе).

Вещественные дифференциалы $d \zeta:=\left(d \zeta_{1}, \ldots, d \zeta_{g}\right)^{\mathrm{t}}$ с обычной $A$-нормировкой

$$
\int_{A_{s}} d \zeta_{j}=\delta_{j s}
$$

связаны с мнимыми дифференциалами $d \boldsymbol{\omega}:=\left(d \omega_{1}, \ldots, d \omega_{g}\right)^{\mathrm{t}}$ следующим образом:

$$
i \Pi d \zeta=d \omega .
$$

3.2.3. Мероморфные дифференциалы. Для построения распределений намагниченности с сингулярностями нам нужны дифференциалы с простыми полюсами. Пусть $W_{p q}$ - гармоническая функция в области $\Omega$ с ровно двумя логарифмическими особенностями противоположного знака в следующих точках: $p$ из замыкания области $\Omega$ и $q$ из ее внутренности. Эта функция удовлетворяет однородному краевому условию Неймана:

$$
\begin{gathered}
W_{p q}(z)=m(p) \ln |z-p|-\ln |z-q|+\text { гармоническая функция, }\left.\quad \frac{\partial W_{p q}(z)}{\partial n}\right|_{z \in \partial \Omega}=0, \\
m(p)= \begin{cases}1, & p \in \operatorname{Int} \Omega, \\
2, & p \in \partial \Omega .\end{cases}
\end{gathered}
$$


Пусть $H_{p q}(z)$ - (многозначная в области) сопряженная к $W_{p q}(z)$ гармоническая функция, тогда дифференциал $d \eta_{p q}:=d\left(W_{p q}+i H_{p q}\right)$ однозначный и вещественный на границе области $\Omega$. Его можно мероморфно продолжить на замкнутую поверхность $\mathcal{D} \Omega$ с помощью действия отражения как вещественный дифференциал:

$$
\tau \cdot d \eta_{p q}=d \eta_{p q}
$$

который будет удовлетворять условию $A$-нормировки $\int_{A_{s}} d \eta_{p q}=0, s=0, \ldots, g$ (интеграл берется в смысле главного значения, если полюс $p$ лежит на вещественном овале).

3.3. Ограничения на нули и полюсы. Здесь мы исследуем ограничения на положения особенностей намагниченности в многосвязном наноэлементе, описываемой вещественным дифференциалом, аналогичные тем, что имеются для кольца.

Отражение $\tau$ естественно действует на дивизорах - конечных формальных суммах точек $p \in \mathcal{D} \Omega$ с целыми коэффициентами:

$$
D=\sum_{p} c(p) \cdot p \rightarrow \tau D=\sum_{p} c(p) \cdot \tau p .
$$

Для каждого зеркально-симметричного дивизора $D=\tau \cdot D$, например дивизора $(d \omega)$ нулей и полюсов вещественного дифференциала $d \omega$, рассмотрим его приведенныц дивизор $D / \tau$ с носителем в замыкании плоской области $\Omega$. Внутренние точки $D / \tau$ наследуют кратность из $D$, а граничные точки имеют половину последней и могут иметь полуцелый вес. Симметричный дивизор равен сумме своего приведенного и его отражения. Всякий (в том числе приведенный) дивизор $D$ естественно разлагается на положительную и отрицательную части: $D=D^{+}-D^{-}, D^{ \pm} \geqslant 0$.

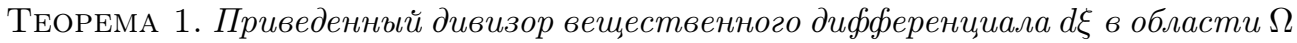
полностью характеризуется тем, что дивизор $D:=(d \xi) / \tau-(d \omega) / \tau=: \sum_{p} c(p) \cdot p$, где $d \omega \neq 0$ - любой фиксированный вещественный дифференциал, удовлетворяет трем условиям:

$$
\begin{gathered}
\operatorname{deg}(D):=\sum_{p} c(p)=0 ; \\
\operatorname{deg}\left(D \mid A_{s}\right):=\sum_{p \in A_{s}} c(p) \in \mathbb{Z}, \quad s=0, \ldots, g ; \\
\left(2 \sum_{p} c(p) \cdot \mathbf{H}(p):=\right) \quad 2 \int_{D^{+}}^{D^{-}} d \mathbf{H} \in \Pi \mathbb{Z}^{g},
\end{gathered}
$$

где $d \mathbf{H}(z):=\left(d H_{1}, \ldots, d H_{g}\right)^{\mathrm{t}}$ и интеграл с дивизорами в пределах интегрирования понимается как сумма интегралов между точками с одинаковым весом, умноженных на этот вес (некоторые внутренние точки можно расщепить на половинки, если это необходимо).

Вещественный дифференииал с заданным приведенным дивизором единственен с точностью до умножения на вещественное число и задается формулой

$$
d \xi(z):=d \omega(z) \exp \left(\int_{z_{0}}^{z} \sum_{p} c(p) d \eta_{p q}\right)
$$


в которой $z_{0} \in A_{0}, q$ - любая внутренняя точка области $\Omega, c(p)$ - полущелая кратность точки р в приведенном дивизоре D, удовлетворяющем условиям (11)-(13).

ЗАмечание 1. Вещественные решеточные условия (13) не зависят от выбора вспомогательного дифференциала $d \omega$, разбиения дивизоров в пределах интегрирования на пары точек и выбора соединяющих их путей интегрирования, лежащих в области $\Omega$.

Пример 4. Определим ограничения на положения $D$ особенностей - (полу)вихрей и седел - в топологических штанах. Используя конформную инвариантность вещественных дифференциалов, конформно отобразим штаны на расширенную плоскость с тремя разделенными щелями, как в примере 2. Дубль Шоттки штанов теперь имеет представление (6) с отражением $\tau(z, w)=(\bar{z},-\bar{w})$. Вспомогательный вещественный голоморфный дифференциал в этой модели имеет вид $d \omega=$ $(i(z-c) / w) d z$ с вещественным $c$. Мы используем следующий симплектический базис циклов: $A_{1}, A_{2} ; B_{1}, B_{2}$ - это обратные образы отрезков $\left[e_{1}, e_{2}\right],\left[e_{5}, e_{6}\right] ;\left[e_{2}, e_{3}\right]$, $\left[e_{4}, e_{5}\right]$ при проекции $(z, w) \rightarrow z$, с подходящей ориентацией. Базис мнимых голоморфных дифференциалов имеет вид $d \omega_{s}=\left(a_{s}\left(z-c_{s}\right) / w\right) d z$, где вещественные коэффициенты $a_{s}$ и нули $c_{1} \in\left[e_{4}, e_{5}\right], c_{2} \in\left[e_{2}, e_{3}\right]$ можно найти из условий нормировки $\int_{B_{s}} d \omega_{j}=-2 \delta_{j s}$. Теперь ограничения (13) на положения нулей $D^{+}$и полюсов $D^{-}$ вещественного дифференциала приобретают вид

$$
2 \operatorname{Im} \int_{D^{+}}^{D_{-}+c} d \boldsymbol{\omega} \in \Pi \mathbb{Z}^{2} .
$$

Последнее условие не зависит от вещественного $c$ : если $w^{2}(c)>0$, то приращение величины $c$ приводит к вещественному приращению последнего интеграла; если же $w^{2}(c)<0$, тогда $c$ представляет пару полуточек на границе штанов, так что приращение значения $c$ не меняет значения всего интеграла.

ДокАЗАТЕЛЬСтво тЕОРЕмы 1 [2], [3], [15]. Зафиксируем вспомогательный вещественный дифференциал $d \omega$, скажем голоморфный дифференциал $d \zeta_{s}$ или $i d \omega_{s}$. Отношение двух вещественных дифференциалов - это вещественная функция: $h(z)=$ $d \xi(z) / d \omega(z)$. Обозначим через $D=\sum_{p} c(p) \cdot p$ приведенный дивизор функции $h(z)$ и проверим три свойства (11)-(13). Первые два из них очевидны: $0=\operatorname{deg}(h)=$ $2 \operatorname{deg} D ;$ на каждой граничной компоненте вещественная функция $h(u)$ изменяет знак в четном числе точек $p$ - своих нулях и полюсах нечетной кратности, т. е. ровно там, где $c(p) \in 1 / 2+\mathbb{Z}$.

Сосредоточимся теперь на условии (13). Для всякой внутренней точки $q \in \Omega$ справедливо следующее представление:

$$
\frac{d h}{h}=\sum_{p} c(p) d \eta_{p q},
$$

в котором $c(p) \in \mathbb{Z} / 2$ - это кратность точки $p$ в приведенном дивизоре функции $h(z)$. В самом деле, обе части равенства имеют одинаковые особенности и интегралы по всем граничным компонентам (понимаемые, быть может, в смысле главного значения) $A_{s}$ исчезают. Справедлива цепочка векторных равенств, в которой В означает 
набор $B$-циклов поверхности $\mathcal{D} \omega$ :

$$
\begin{aligned}
2 \pi i \mathbb{Z}^{g} & \ni \int_{\mathbf{B}} \frac{d h}{h}=\sum_{p} c(p) \int_{\mathbf{B}} d \eta_{p q} \stackrel{*}{=} 2 \pi i \sum_{p} 2 c(p) \operatorname{Re}\left[\int_{q}^{p} d \boldsymbol{\zeta}\right]= \\
& =2 \pi i \sum_{p} 2 c(p) \operatorname{Im}\left[\Pi^{-1} \int_{q}^{p} d \boldsymbol{\omega}\right]=2 \pi i \sum_{p} 2 c(p) \Pi^{-1} \int_{q}^{p} d \mathbf{H}
\end{aligned}
$$

где равенство со звездочкой следует из римановых билинейных соотношений (законов взаимности), а все суммы по точкам $p$ берутся по носителю приведенного дивизора $D$. Мы группируем члены с противоположным значением кратности $c(p)$ в последней сумме и получаем в точности условие (13).

Обращение рассуждения. Предположим, что три условия теоремы выполнены для приведенного дивизора $D$. Нужно проверить три утверждения для дифференциала (14).

1. Дифференциал $d \xi$ имеет в области приведенный дивизор в точности $D+(d \omega) / \tau$. В самом деле, $\operatorname{deg} D=0$ и интеграл в (14) не имеет особенности при $z=q$.

2. Дифференциал $d \xi$ однозначен в области. Это следует из $A$-нормированности элементарных сингулярных интегралов $d \eta_{p q}$ и условия (12).

3. Дифференциал вещественный на границе области. Действительно,

$$
2 i \operatorname{Im}\left[\int_{B_{s}^{+}} d \eta_{p q}\right]=\int_{B_{s}} d \eta_{p q},
$$

и сумму последних интегралов по точкам $p$ с их весами можно извлечь из цепочки равенств (15), что вместе с ограничением (13) означает, что экспоненциальный множитель в равенстве (14) вещественный на границе области $\Omega$.

\section{4. ВЕЩЕСТВЕННЫЕ ДИФФЕРЕНЦИАЛЫ ПРИМА И НЕЛОКАЛЬНЫЕ МЕРОНЫ}

Гросс [5] пишет: “Хотя мы и нашли бесконечный класс решений нелинейных уравнений движения, понятно, что это не общее решение". Все опущенные меронные решения будут описаны в текущем разделе. Вспомним (см. раздел 2), что общее локальное решение уравнения (4) с $|w(z)|=1$ вне особенностей намагниченности имеет вид $w(z)=f /|f|$ с голоморфной функцией $f(z) \neq 0$. Эта функция глобально может и не быть однозначной, и все же приводить к однозначному намагничиванию тогда и только тогда, когда $f(z)$ домножается на положительную функцию при обходе ее аргументом особенностей и/или граничных компонент магнитного элемента. Эта положительная функция с необходимостью является (локально) постоянной, что приводит к возникновению монодромии:

$$
\rho: \pi_{1}(\Omega-\operatorname{Supp}(f)) \rightarrow \mathbb{R}^{+} .
$$

(Коммутативная) монодромия в области с конечным числом проколов полностью определяется монодромиями $\rho(p)$ петель, огибающих проколы $p \in \operatorname{Supp}(f)$ против часовой стрелки, а также монодромиями $\rho\left(A_{s}\right)$ ориентированных граничных компонент с единственным соотношением

$$
\prod_{p} \rho(p)=\prod_{s=0}^{g} \rho\left(A_{s}\right) .
$$


Определим вид функции $f(z)$ вблизи изолированной точечной особенности $z=p$ намагниченности. Функция $h(z):=(z-p)^{-i \mu}$ при $2 \pi \mu=\ln (\rho(p))$ имеет ту же локальную монодромию, что и $f$, а значит, функция $f / h$ однозначна вблизи особенности $z=p$. Эта точка не может быть существенно особой для $f / h$, в противном случае мы теряем свойство логарифмической расходимости энергии (см. раздел 2), но она может быть нулем или полюсом конечного порядка. Тем самым мы получаем локальное поведение многозначного дифференциала $d \xi:=d z / f(z)$ вблизи точек его дивизора:

$$
d \xi(z)=(z-p)^{m+i \mu} \phi(z) d z, \quad \phi(z) \in \mathcal{O}^{*}(p), \quad m \in \mathbb{Z}, \quad \mu \in \mathbb{R},
$$

с голоморфной функцией $\phi(z)$, не обращающейся в нуль вблизи точки $p$. Этот дифференциал имеет положительную монодромию и, как и ранее, он вещественный на границе области. Последнее свойство корректно определено даже с учетом монодромии. Подобные объекты называют вещественными дифференциалами Прима [15], [16].

Дивизор $D=(d \xi)$ вещественного дифференциала Прима $d \xi$ имеет комплексные веса $c(p)=m(p)+i \mu(p)$ с (целой в нашем случае) вещественной частью, определяемой ростом дифференциала вблизи особенности $z=p$, и мнимой частью, связанной с положительной локальной монодромией. Такой дивизор остается неизменным при естественном действии отражения $\tau$ на дивизоры с комплексными весами:

$$
D:=\sum_{p} c(p) \cdot p \rightarrow \tau \cdot D:=\sum_{p} \overline{c(p)} \cdot \tau p
$$

в частности, точки вещественных овалов имеют чисто вещественный вес. Для симметричного дивизора с комплексными весами $D=\tau \cdot D$ на дубле области определим его проекцию $D / \tau$ на область $\Omega$ дубля как дивизор с носителем в замыкании области, с сохраненными кратностями внутренних точек и половинными кратностями граничных точек.

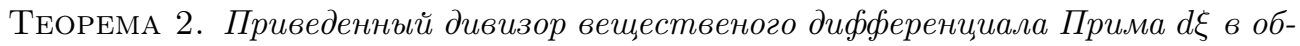
ласти $\Omega$ с заданной монодромией $\rho: \pi_{1}(\Omega-\operatorname{Supp}(d \xi)) \rightarrow \mathbb{R}^{+}$полностъю определяется тем, что дивизор с комплексными весами $D:=(d \xi) / \tau-(d \omega) / \tau:=\sum_{p} c(p) \cdot p$, где $d \omega \neq 0$ - произвольный фиксированный вещественный абелев дифференииал, удовлетворяет четырем условиям:

1) мнимая часть D определена монодромией:

$$
\operatorname{Im} D=-\frac{1}{2 \pi} \sum_{p} \ln \rho(p) \cdot p
$$

2) $\operatorname{deg} \operatorname{Re} D=0$, веса $\operatorname{Re} c(p)$ целье внутри области и полуцелье на ее границе;

3) $\operatorname{deg} \operatorname{Re}\left(D \mid A_{s}\right):=\sum_{p \in A_{s}} \operatorname{Re} c(p) \in \mathbb{Z}, s=0, \ldots, g$;

4) выполнено решеточное условие

$$
2 \operatorname{Im} \sum_{p} c(p) \int_{z_{0}}^{p} d \boldsymbol{\omega}+\frac{1}{\pi} \ln \rho(\mathbf{A}) \in \Pi \mathbb{Z}^{g},
$$

где $\ln \rho(\mathbf{A})-$ вектор $\left(\ln \rho\left(A_{1}\right), \ldots, \ln \rho\left(A_{g}\right)\right)^{\mathrm{t}}, z_{0} \in A_{0}$. 
Вещественный дифференииал Прима восстанавливается по своему дивизору однозначно с точностью до умножения на вещественную постоянную:

$$
d \xi(z)=d \omega(z) \exp \left(\int_{z_{0}}^{z} d \eta+\sum_{s=1}^{g} \ln \rho\left(A_{s}\right) d \zeta_{s}\right),
$$

где $d \eta$ - (вещественный абелев) A-нормированный дифференииал на дубле $\mathcal{D} \Omega$ с простыми полюсами и дивизором вычетов $D+\tau \cdot D$, а приведенный дивизор $D$ удовлетворяет четырем приведенным выше условиям.

ДокАЗАТЕЛЬСТво этой теоремы в основном следует рассуждениям из доказательства теоремы 1 . Отношение $h(z)=d \xi / d \omega-$ это вещественная функция Прима с той же монодромией, что и у $d \xi$ и с приведенным дивизором $D$. Вещественный абелев дифференциал $d \ln h(z)$ можно разложить следующим образом:

$$
d \ln h=d \eta+d \zeta
$$

с $A$-нормированным дифференциалом $d \eta$, имеющим те же особенности, что и $d \ln h$ (теперь мы не можем разложить его на элементарные члены $d \eta_{p q}$, как это сделано в п. 3.2.3, и должны использовать теорему существования [15]), а также вещественный голоморфный дифференциал $d \zeta$, дальнейшее разложение которого по базисным дифференциалам $d \zeta_{s}$ можно найти интегрированием обеих частей последнего равенства по вещественным овалам. Включение

$$
2 \pi i \mathbb{Z}^{g} \ni 2 \operatorname{Im}\left[\int_{\mathbf{B}^{+}} d \ln h\right]=\int_{\mathbf{B}} d \ln h
$$

после подстановки приведенного выше разложения и использования римановых законов взаимности, наконец, приводит к решеточному условию (18) теоремы.

\section{1. Примеры.}

4.1.1. Диск. Перечислим все нелокальные меронные решения в односвязной области. Такую область можно конформно отобразить на полуплоскость, поэтому, не теряя общности, предположим, что $\Omega=\mathbb{H}$. Общий вещественный дифференциал Прима имеет вид $d \xi(z)=h(z) d z$ с вещественной функцией $h(z)$, являющейся произведением нескольких элементарных функций вида

$$
\begin{aligned}
h^{ \pm}(z \mid a, \mu) & =(z-a)^{ \pm 1+i \mu}(z-\bar{a})^{ \pm 1-i \mu}, & & a \in \mathbb{H}, \quad \mu \in \mathbb{R}, \\
h^{ \pm}(z \mid c) & =(z-c)^{ \pm 1}, & & c \in \mathbb{R},
\end{aligned}
$$

с различными параметрами $\pm, a, \mu, c$.

4.1.2. Кольцо. Здесь мы перечислим в терминах эллиптических тета-функций все нелокальные меронные решения в кольце, представленном как фактор полосы $0 \leqslant \operatorname{Re} u<1 / 2$ по группе сдвигов: такая модель рассматривалась в примере 3 (см. п. 3.1). Отображение на концентрическое кольцо дается показательной функцией $z(u)=e^{2 \pi u / T}, T>0$. Общее решение $d \xi(u)=i h(u) d u$ можно всегда разложить на элементарные: 1) без особенностей (голоморфное); 2) внутренние вихрь и антивихрь; 3) один внутренний антивихрь и два полувихря на границе. Три упомянутых случая соответствуют следующему выбору вещественной мероморфной функции $h(u)$ в полосе. 
1. Решение без особенностей в кольце соответствует функции

$$
h(u \mid m)=e^{2 \pi i m u}, \quad m \in \mathbb{Z} .
$$

Интегральные кривые поля намагниченности $w(z)$ при этом образуют $|m|$ вложенных слоений Риба в концентрическом кольце с внутренними магнитными доменными стенками, представленными предельными циклами поля намагниченности. Конфигурации такого вида наблюдались в экспериментах.

2. Решение с вихрем и седлом в двух внутренних точках кольца соответствует функции

$$
h\left(u \mid a_{ \pm}, \mu_{ \pm}\right)=\frac{\theta_{1}^{1+i \mu_{+}}\left(u-a_{+}\right) \theta_{1}^{1-i \mu_{+}}\left(u+\overline{a_{+}}\right)}{\theta_{1}^{1+i \mu_{-}}\left(u-a_{-}\right) \theta_{1}^{1-i \mu_{-}}\left(u+\overline{a_{-}}\right)}, \quad \mu_{ \pm} \in \mathbb{R} ; \quad 0<\operatorname{Re} a_{ \pm}<\frac{1}{2} .
$$

Приведенный дивизор $D=\left(1+i \mu_{+}\right) \cdot z\left(a_{+}\right)-\left(1+i \mu_{-}\right) \cdot z\left(a_{-}\right)$в кольце определяет монодромию решения.

3. Конфигурация намагниченности включает антивихрь (седло) во внутренности кольца и два полувихря на одной из его границ при

$$
\begin{gathered}
h\left(u \mid a, a_{1}, a_{2}, \mu\right)=\frac{\theta_{1}^{1+i \mu}(u-a) \theta_{1}^{1-i \mu}(u+\bar{a})}{\theta_{1}\left(u-a_{1}\right) \theta_{1}\left(u-a_{2}\right)} \\
\mu \in \mathbb{R} ; \quad \operatorname{Re} a_{1}=\operatorname{Re} a_{2} \in\left\{0, \frac{1}{2}\right\} ; \quad 0<\operatorname{Re} a<\frac{1}{2} .
\end{gathered}
$$

Приведенный дивизор вещественного дифференциала Прима $d \xi=i h(u) d u$, перенесенного в кольцо, есть

$$
D=(1+i \mu) \cdot z(a)-\frac{1}{2} \cdot z\left(a_{1}\right)-\frac{1}{2} \cdot z\left(a_{2}\right) .
$$

Во всех трех случаях проверяется, что функция $h(u)$ имеет период 1 , вещественна на границе полосы и приобретает постоянный положительный множитель при сдвиге $u \rightarrow u+i T$.

\section{5. ЗАКЛЮЧЕНИЕ}

Статья посвящена математическим аспектам описания топологически заряженных магнитных текстур [1], [6] в многосвязных планарных магнитных наноэлементах. Новые многовихревые конфигурации - точные решения нелинейной граничной задачи, описывающей микромагнитные состояния в модели Метлова, - были введены в работе [10] в терминах так называемых вещественных мероморфных дифференциалов на замкнутой римановой поверхности, дубле Шоттки первоначальной области. Этот подход подчеркивает конформную инвариантность задачи и позволяет дать эффективные формулы для решений в алгебраических терминах, а также на языке тета-функций [12], [14], функций Шоттки [17] и главной формы Шоттки-Клейна [10], [14], [18]. Подход позволил явно перечислить все магнитные текстуры, следующие из модели [1], [6], в одно-, двух- и трехсвязных областях. Мы установили полный набор ограничений на число и положения возможных особенностей намагниченности для области произвольной связности. Это распространяет наши прежние результаты об ограничениях на положения топологических особенностей в кольце [4], которые были проверены в эксперименте. Ограничения на положения особенностей намагниченности могут быть интересны и для приложений: 
в спинтронике вихревые конфигурации являются носителями информации и возникает возможость проверить, не было ли ошибок при ее считывании.

В разделе 4 используется свойство меронного поля намагниченности оставаться неизменными при растяжении порождающей его мероморфной функции. Математически это приводит к нелокальным объектам, дифференциалам Прима на дубле планарной области. Эти решения обладают новыми скрытыми степенями свободы локальной и глобальной монодромией, которые можно использовать как параметры Ритца для минимизации следующих членов в иерархии энергий или как динамические переменные в адиабатическом подходе.

Благодарности. Своему знакомству с увлекательным миром магнитных явлений автор обязан Константину Метлову из Донецкого физико-технического института, который также сформулировал несколько физически значимых математических задач.

\section{Список литературы}

[1] K. L. Metlov, "Magnetization patterns in ferromagnetic nanoelements as functions of complex variable", Phys. Rev. Lett., 105:10 (2010), 107201, 4 pp.

[2] M. Schiffer, D. C. Spencer, Functionals of Finite Riemann Surfaces, Princeton Univ. Press, Princeton, NJ, 1954.

[3] N. L. Alling, N. Greenleaf, Foundations of the Theory of Klein Surfaces, Lecture Notes in Mathematics, 219, Springer, Berlin, 1971.

[4] A. B. Bogatyrev, K. L. Metlov, "Topological constraints on positions of magnetic solitons in multiply-connected planar magnetic nanoelements", Phys. Rev. B, 95:2 (2017), 024403, 5 pp.; arXiv: 1609.02509.

[5] D. J. Gross, "Meron configurations in the two-dimensional $\mathrm{O}(3) \sigma$-model", Nucl. Phys. B, 132:5 (1978), 439-456.

[6] K. L. Metlov, Two-dimensional topological solitons in soft ferromagnetic cylinders, arXiv: cond-mat/0102311.

[7] Ф. Д. Гахов, Краевые задачи, Наука, М., 1977.

[8] A. Aharoni, Introduction to the Theory of Ferromagnetism, Oxford Univ. Press, Oxford, 1996.

[9] А.А. Белавин, А. М. Поляков, "Метастабильные состояния двумерного изотропного ферромагнетика", Писъма в ЖЭТФ, 22:10 (1975), 503-506.

[10] A. B. Bogatyrev, K. L. Metlov, "Magnetic states in multiply-connected flat nano-elements", ФHT, 41:10 (2015), 984-988, arXiv: 1504.01162.

[11] А.Б. Богатырёв, “Элементарная конструкция штребелевых дифференциалов", $M a-$ тем. заметки, 91:1 (2012), 143-146.

[12] Н. И. Ахиезер, Элементы теории эллиптических функиий, Наука, М., 1970.

[13] Ф. Гриффитс, Дж. Харрис, Приниипь алгебраической геометрии, т. 1, 2, Мир, М., 1982.

[14] Д. Мамфорд, Лекции о тэта-функции, Мир, М., 1988.

[15] H. M. Farkas, I. Kra, Riemann Surfaces, Graduate Texts in Mathematics, 71, Springer, New York, Berlin, 1980.

[16] В. В. Чуешев, Мультипликативные функиии и дифференциалы Прима на переменной римановой поверхности, КемГУ, Кемерово, 2003.

[17] A. Bogatyrev, "Computations in moduli spaces", Computat. Meth. Funct. Theory, 7:2 (2007), 309-324.

[18] A. Bogatyrev, "Prime form and Schottky model", Computat. Meth. Funct. Theory, 9:1 (2009), 47-55. 